\title{
En préambule aux deux entretiens croisés sur l'impact du nucléaire
}

François Graner, physicien, CNRS, et Stefano Matthias Panebianco, physicien, CEA Éditeurs du dossier

L'idée que l'opinion publique se fait du nucléaire civil et l'évolution de cette appréciation au gré des incidents, plus ou moins graves, et des articles de presse, est à l'image de la variété des positions, pour la plupart contradictoires, qui traversent la communauté scientifique, les politiques et de manière générale tous les acteurs de la société. Cette polarisation des avis est une caractéristique constante du débat sur le nucléaire. Pour autant, les opposants comme les partisans du nucléaire proposent des arguments qui méritent d'être présentés, analysés de façon critique, et évalués.

Comme pour toute activité humaine technologique de grande échelle, complexe et diversifiée, l'évaluation de l'impact environnemental du nucléaire civil doit prendre en compte toutes les phases concernées par les nombreux procédés industriels qui caractérisent la filière. Les risques envisageables sont de nature très différente, sur des territoires variés, d'autant que malgré les dispositions prises lors de la conception et de l'exploitation des installations, un accident, qu'il soit lié à un évènement naturel, une faute humaine ou une malveillance, ne peut pas être exclu. Dans le cas du nucléaire, les différents aspects liés à la production d'énergie vont des conditions d'extraction minière du combustible jusqu'au devenir des combustibles usés, avec des spécificités qui sont au centre des débats sur cette technologie : possibilités d'irradiation, de contamination, de pollution chimique ou d'explosion.

Dans cet état d'esprit, nous avons voulu interroger des scientifiques partisans et opposés au nucléaire civil sur l'impact environnemental et sanitaire de la filière nucléaire civile, en situation non accidentelle ${ }^{(a)}$. Pour cela, nous avons sollicité Claude Stéphan, physicien nucléaire qui a beaucoup écrit sur la filière nucléaire civile et qui en est plutôt un partisan, et Pierre Barbey, biologiste de l'Université de Caen, directeur des Installations de Mise en Oeuvre et de GEstion des Radioéléments (IMOGERE), qui a pris des positions critiques sur les émissions des centrales. Dans le même état d'esprit, nous avons sollicité Jean-Christophe Gariel de l'Institut de Radioprotection et de Sureté Nucléaire (IRSN) et la juriste Sophia Majnoni d'Intignano, anciennement très active au sein de Greenpeace, pour commenter la prévention et la gestion d'un éventuel accident ${ }^{(b)}$.

Dans chacun des deux cas, les auteurs sollicités ont développé des arguments importants et complémentaires. Nous vous en proposons une sélection certes arbitraire, la plus synthétique possible. Les deux articles qui vont suivre n'engagent que notre responsabilité et non celles des personnes interrogées. Ils constituent un exemple du débat qui traverse le milieu scientifique et la société toute entière, débat où les domaines considérés sont

a. Voir page 19 l'entretien croisé avec C. Stéphan et P. Barbey.

b. Voir page 22 l'entretien croisé avec J.-C. Gariel et S. Majnoni d'Intignano. aussi variés que la physique et l'ingénierie nucléaire, l'économie, la santé, et qui va jusqu'à des considérations sociologiques et morales.

Pour illustrer plus en détail ce large éventail de sujets, ces deux entretiens croisés sont suivis d'autres textes plus spécialisés traitant du rôle des associations et de la sous-traitance dans le milieu du nucléaire, ainsi que des couts d'exploitation de la filière. 